\title{
Effect of sequential respiratory training on patients with stable chronic obstructive pulmonary disease: a randomized controlled clinical trial
}

\author{
Xiaobing Wang ${ }^{1 *}$, An Guo ${ }^{2 *}$, Honggang Wang ${ }^{2}$, Wei Huang ${ }^{1}$ and Mingxing Ding ${ }^{3 *}$ \\ ${ }^{1}$ Department of Rehabilitation Medicine, School of Medicine, Jinhua Polytechnic, Jinhua 321007, Zhejiang, China \\ ${ }^{2}$ Department of Respiration, Jinhua People's Hospital, Jinhua 321000, Zhejiang, China \\ ${ }^{3}$ Medical Molecular Biology Laboratory, School of Medicine, Jinhua Polytechnic, Jinhua 321007, Zhejiang, China \\ \#Contributed equally to this work
}

\begin{abstract}
Objective: To explore the effect of sequential respiratory training rehabilitation methods on the pulmonary function of patients with stable chronic obstructive pulmonary disease.

Methods: This is a randomized controlled clinical trial. A total of 200 cases of stable chronic obstructive pulmonary disease patients were recruited from June 2014 to May 2015 in the respiratory medicine clinic, which were randomly divided into sequential breathing training group and control group. Forced expiratory volume in the first second (FEVI\%), British Medical Research Council (mMRC) questionnaire, Chronic Obstructive Pulmonary Disease Assessment Test (CAT) score, the number of acute exacerbation frequency were and 6-minute walk test (6MWT) were assessed after treatment for 3, 6 and 12 months.

Results: During the 12-month follow-up period, a total of 170 patients had complete follow-up information. There were 85 patients both in sequential breathing training group and control group. In the experimental group, there were 59 males and 26 females, with an average age of (65.02 \pm 7.84$)$ years. In the control group, there were 64 males and 21 females, with an average age of $(64.96 \pm 7.02)$ years. After treatment for 3, 6 and 12 months, FEVI\%, mMRC score, CAT score, the number of acute exacerbation frequency and 6MWT were all significantly improved in the experimental group than control group ( $\mathrm{p}<0.05$ ).
\end{abstract}

Conclusion: Sequential breathing training therapy can better improve the pulmonary function of stable chronic obstructive pulmonary disease patients and distinctly reduce the incidence of acute exacerbation of chronic obstructive pulmonary disease, which provides a basis for clinical rehabilitation.

Trial registration: This study was registered in the Chinese Clinical Trials Registry Platform (ChiCTR-OPC-14005290).

Abbreviations: mMRC: British Medical Research Council; CAT: COPD Assessment Test; 6MWT: 6-minute walk test; SD: standard deviation; BMI: body mass index.

\section{Introduction}

Chronic obstructive pulmonary disease is one of the most common respiratory system diseases worldwide (approximately $11.7 \%$ prevalence), characterized by small airway obstruction and emphysema [1]. The most common risk factors for chronic obstructive pulmonary disease are smoking and other environmental factors [2]. The main method of controlling stable chronic obstructive pulmonary disease is to inhale long-acting bronchodilators [3]. In addition to quitting smoking, there are currently no effective methods to slow down the progress of chronic obstructive pulmonary disease [3,4]. Thus, there is a need to develop novel therapies for chronic obstructive pulmonary disease [5].

Pulmonary rehabilitation training has been considered as the main recommended therapy in the non-pharmacological treatment of chronic obstructive pulmonary disease prevention and treatment $[6,7]$. The methods involved mainly include exercise therapy, social psychological support, behavioral intervention, and publicity and education, and the core is respiratory training [8-10]. Studies have shown that lung rehabilitation can improve the exercise capacity and life quality of chronic obstructive pulmonary disease patients, including dyspnea, fatigue, and mood [11-13]. Its curative effect evaluation index is relatively comprehensive, basically covering the control of symptoms, daily activity ability, exercise ability, hospital admission rate and utilization of social health resources [14,15]. Nevertheless, these studies also have problems such as incomplete rehabilitation methods and lack of systemicity [16]. Also, there is still a big gap between the current status of domestic lung rehabilitation and the multidisciplinary team of lung rehabilitation. In this study, we proposed a practical, effective, and convenient method for patient application, effective sequential respiratory rehabilitation treatment of chronic obstructive pulmonary disease patients and evaluation of rehabilitation efficacy, which can provide a basis for better rehabilitation treatment for clinical chronic obstructive pulmonary disease patients.

${ }^{\star}$ Correspondence to: Mingxing Ding, Medical Molecular Biology Laboratory, School of Medicine, Jinhua Polytechnic, No.1188 Wuzhou Street, Jinhua 321007, Zhejiang, China, E-mail: mtd5tc@163.com

Key words: stable chronic obstructive pulmonary disease; sequential respiratory training; 6-minute walk test; pulmonary function; respiratory rehabilitation

Received: June 07, 2020; Accepted: June 16, 2020; Published: June 19, 2020 


\section{Materials and methods}

\section{Participates}

According to the diagnosis and classification standards of the revised guidelines of the 2014 Global Initiative for Chronic Obstructive Pulmonary Disease, a total of 200 chronic obstructive pulmonary disease patients (aged 45-65 years) with pulmonary grades II-III grade and in stable phase were included in this study between June 2014 to May 2016 in the Jinhua People's Hospital. The exclusion criteria were as follows: patients with hypertension, diabetes, coronary heart disease, severe bronchial asthma, cardiomyopathy, malignant tumors, allergic disease, bronchiectasis, severe liver and kidney disease and other infectious, trauma, hematopoietic system diseases, nervous system diseases, mental system diseases, autoimmune system diseases, active tuberculosis, gout, stroke, pregnant women, lactating women and so on. This study was approved by the ethics committee of School of Medicine, Jinhua Polytechnic (2014035). This study was registered in the Chinese Clinical Trials Registry Platform (ChiCTR-OPC-14005290).

\section{Treatment and assessment}

All patients were comprehensively evaluated before enrollment, including CAT questionnaire, 6-minute walk test, pulmonary ventilation function assessment, and acute exacerbation risk assessment. These patients were randomly divided into sequential breathing training group (experimental group) and control group. The sequential breathing training group was given sequential breathing training based on conventional medicine, while the control group was given single breathing training based on conventional medicine. The CAT questionnaire score, 6-minute walking distance test, acute exacerbation risk assessment, and pulmonary ventilation function assessment were performed on the two groups of patients at 3,6, and 12 months after the experiment. During the 12-month follow-up period, the patient returned to the hospital for further treatment if the symptoms worsened again. During the follow-up period, the frequency of acute chronic obstructive pulmonary disease exacerbation of the two groups of patients was counted. Finally, a total of 170 patients completed follow-up and 30 were lost to follow-up. The indicators monitored during training were as follows: target heart rate; fingertip blood oxygen $\mathrm{SpO}_{2}>90 \%$; blood pressure $<200 / 100 \mathrm{mmHg}$; Borg index $<5$ (more severe shortness of breath). The frequency of sequential breathing training was 3 times a week and lasted for 12 weeks.

Sequential breathing training methods were as follows: (1) Abdominal breathing: patient was taken a supine position; hip and knee joints were slightly bent, and the whole body was in a comfortable position. Patient put his right hand on his abdomen and his left hand on his chest. He took a deep breath, concentrated his thoughts, and allowed the patient to feel the hand change during inhalation and exhalation. The training time was 15 minutes, twice a day. (2) Contract lips after reaching the previous goal: during abdominal breathing, patient inhaled and exhaled with the nose and closed the lips slightly, and gently exhaled the gas. The ratio of inhalation to exhalation was from 1:2. The goal of exhalation ratio was 1: 4 , the training time was 15 minutes, twice a day. (3) After reaching the previous goal, the abdominal weight-bearing method was used to strengthen the training of respiratory muscles: the abdominal breath was used to resist the abdominal heavy objects. The patient was still knee flexion and supine position, added a small sandbag on the upper abdomen, the weight of the sandbag was to target the load that could complete 10 abdominal breaths, and gradually increased the amount, 10 times per process and lasted for 15 minutes twice a day. (4) After reaching the previous goal, upper limb muscle training was performed: lifting weights (small sandbags 250-500g) each group lift 15 times, 3 groups each time, twice a day. (5) Lower limb muscle training after reaching the previous goal: level walking training for 15 minutes, twice a day.

\section{Statistical analysis}

All statistical analysis was implemented using SPSS21.0 software. Normally distributed data were expressed as mean \pm standard deviation (SD), and non-normally distributed data were presented as median (interquartile range). Normally distributed data were compared with the student's t test, while non-normally distributed data were compared with the non-parametric test. Count data was evaluated using $\chi^{2}$ test. $\mathrm{P}<0.05$ indicated statistical significance.

\section{Results}

\section{General characteristics of participants}

There were 85 patients in sequential breathing training group (experimental group), including 59 males and 26 females, with an average age of $(65.02 \pm 7.84)$ years. There were 85 patients in the control group, including 64 males and 21 females, with an average age of $(64.96 \pm 7.02)$ years. Before the experiment, there were no statistical differences in the average age, gender, body mass index (BMI), pulmonary function assessment (FEV1\% predicted value), CAT score, mMRC score, 6-minute walk test (6MWT), and the number of acute exacerbations within one year between the two groups (Table 1).

\section{Comparison of pulmonary function between the experimental and control groups before and after treatment}

There was no significant difference in FEVI\% between experimental and control groups before treatment. After 3 months, 6 months and 12 months of treatment, the FEVI of patients in experimental or control groups was significantly higher compared to before treatment. Compared with the control group, patients in the sequential breathing training group had significantly higher FEVI\% after treatment for 3 months, 6 months and 12 months in Table 2. Thus, sequential breathing training could improve pulmonary function of patients with chronic obstructive pulmonary disease.

\section{Comparison of 6MWD between the experimental and control groups before and after treatment}

Before treatment, there was no significant difference in the 6MWD between experimental and control groups. After treatment for 3, 6 and 12 months, the 6MWD of the two groups of patients was significantly higher compared to before treatment. Compared with the control group after treatment, the $6 \mathrm{MWD}$ of the sequential breathing training group was significantly improved, as listed in Table 3.

\section{Comparison of CAT and mMRC scores of between the experimental and control groups before and after treatment.}

The CAT and mMRC scores of the experimental and control groups of patients before and after treatment were compared. The results showed that the CAT scores of the two groups of patients were distinctly decreased with time. Compared with before treatment, there were statistical significances in CAT scores after treatment for 3, 6 and 12 months both in the two groups (Figure 1). The experimental group had a significantly lower CAT scores than the control group. Moreover, both in the two groups after treatment for 3, 6 and 12 months had notably higher mMRC scores than before treatment. There were significantly higher mMRC scores in experimental group compared to control group after treatment for 3, 6 and 12 months (Figure 2). 
Table 1. General characteristics of participants in the experimental and control groups

\begin{tabular}{|c|c|c|c|}
\hline Characteristics & Experimental group $(\mathrm{n}=\mathbf{8 5})$ & Control group $(n=85)$ & P-value \\
\hline Sex & & & $>0.9999$ \\
\hline Male (\%) & $59(69.41)$ & $64(75.29)$ & \\
\hline Female (\%) & $26(30.59)$ & $21(24.71)$ & \\
\hline $\operatorname{BMI}\left(\mathrm{x} \pm \mathrm{s}, \mathrm{kg} / \mathrm{m}^{2}\right)$ & $21.55 \pm 3.17$ & $21.13 \pm 2.26$ & 0.3213 \\
\hline FEVI $(\mathrm{x} \pm \mathrm{s}, \%)$ & $58.70 \pm 6.12$ & $58.43 \pm 5.15$ & 0.7560 \\
\hline CAT score $(\mathrm{x} \pm \mathrm{s})$ & $27.40 \pm 2.61$ & $27.32 \pm 3.13$ & 0.8566 \\
\hline mMRC score $(\mathrm{x} \pm \mathrm{s})$ & $2.32 \pm 0.10$ & $2.25 \pm 0.21$ & 0.0062 \\
\hline $6 \mathrm{MWT}(\mathrm{x} \pm \mathrm{s}, \mathrm{m})$ & $278.25 \pm 8.13$ & $275.95 \pm 9.34$ & 0.0887 \\
\hline $\begin{array}{l}\text { Number of acute exacerbations within } 1 \text { year } \\
(\mathrm{x} \pm \mathrm{s})\end{array}$ & $1.96 \pm 0.20$ & $1.85 \pm 0.40$ & 0.0791 \\
\hline
\end{tabular}

Table 2. Comparison of pulmonary function between the experimental and control groups before and after treatment

\begin{tabular}{|l|c|c|c|}
\hline \multirow{2}{*}{} & \multicolumn{2}{|c|}{ FEVI } & Control group (n=85) \\
\cline { 2 - 4 } & Experimental group (n=85) & $1.03 \pm 0.19$ \\
\hline Before treatment & $1.01 \pm 0.08$ & $1.07 \pm 0.21$ \\
\hline 3-months treatment & $1.15 \pm 0.23$ & $1.12 \pm 0.22$ \\
\hline 6-months treatment & $1.32 \pm 0.26$ & $1.14 \pm 0.23$ & 0.75 \\
\hline P-value & $1.37 \pm 0.27$ & 0.007 \\
\hline
\end{tabular}

Table 3. Comparison of 6MWD between the experimental and control groups before and after treatment

\begin{tabular}{|l|c|c|c|}
\hline \multirow{2}{*}{ Before treatment } & \multicolumn{2}{|c|}{ 6MWD } & P-value \\
\cline { 2 - 4 } & Experimental group (n=85) & $377.19 \pm 24.61$ \\
\hline 3-months treatment & $375.07 \pm 21.72$ & $392.93 \pm 28.34$ \\
\hline 6-months treatment & $409.07 \pm 33.17$ & $397.49 \pm 26.95$ & 0.65 \\
\hline 12-months treatment & $439.52 \pm 32.32$ & $402.15 \pm 24.06$ \\
\hline P-value & $467.30 \pm 27.98$ & $<0.01$ & $<0.01$ \\
\hline
\end{tabular}

CAT

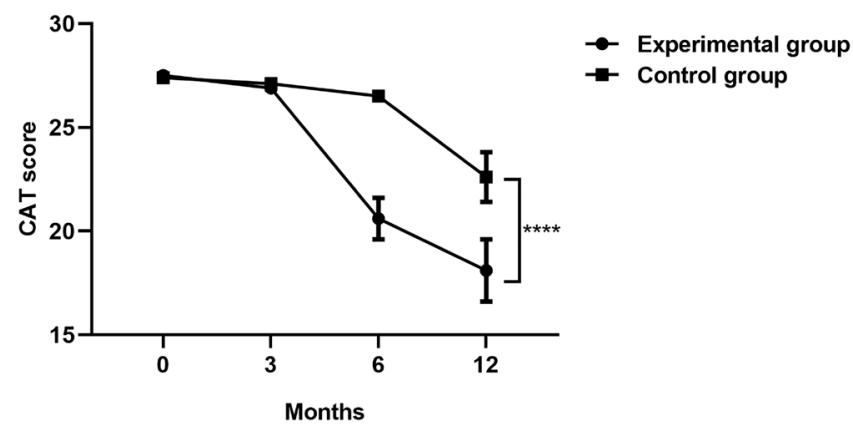

Figure 1. Comparison of CAT scores of between the experimental and control groups before and after treatment

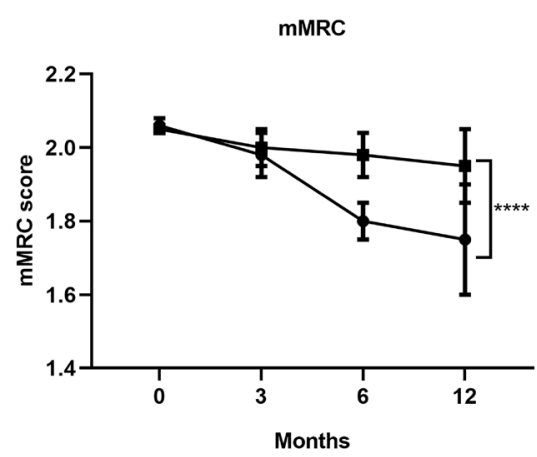

$\rightarrow$ Experimental group Control group

Figure 2. Comparison of mMRC scores of between the experimental and control groups before and after treatment

\section{Discussion}

Pulmonary rehabilitation has gradually been utilized to treat chronic obstructive pulmonary disease patients with dyspnea and exercise endurance, which has become one of the important means for the treatment of chronic obstructive pulmonary disease in the stable period [17-19]. The clinical application of lung rehabilitation has achieved considerable results [20]. For example, studies have found that lung rehabilitation can not only delay chronic obstructive pulmonary disease patients' illness and relieve symptoms of dyspnea, but also restore lung function and improve motor function [21-23]. However, at present, a unified standard for rehabilitation therapy has been not established. Moreover, physicians engaged in respiratory specialties have not yet recognized the role of rehabilitation therapy in improving lung function and quality of life in patients with chronic obstructive pulmonary disease [24]. In this study, we performed a randomized controlled clinical trial to investigate the effect of sequential respiratory training on stable chronic obstructive pulmonary disease. Our results suggested that sequential respiratory training can significantly improve FEVI\%, mMRC score, CAT score, the number of acute exacerbation frequency and 6MWT for stable chronic obstructive pulmonary disease. Thus, sequential respiratory training could become a potential therapy for pulmonary rehabilitation.

Many studies have confirmed that mMRC and CAT can well reveal the characteristics of chronic obstructive pulmonary disease [25-27]. However, mMRC score is a one-dimensional measurement that can only quantify dyspnea. The CAT score is a multi-dimensional method that can evaluate 8 items; not only dyspnea, but also other symptoms and health conditions. Moreover, it has the advantage of being easy to implement and can be associated with clinically important variables 
such as FEVI\% and acute exacerbation. Therefore, utilization of CAT to evaluate the quality of life of patients with chronic obstructive pulmonary disease can help doctors judge the patient's condition, which is scientific and reliable [28-30]. In our study, we compared the difference between the effects of sequential respiratory training and basic conventional methods on the lung function and quality of life scores of patients with stable chronic obstructive pulmonary disease. We selected CAT and mMRC scores as evaluation criteria for chronic obstructive pulmonary disease. The results showed that the sequential breathing training method can effectively improve the FEVI\% level of lung function in chronic obstructive pulmonary disease patients after treatment for 3, 6 and 12 months. Previous studies have demonstrated the correlation between CAT and 6MWT [31]. 6MWT has been considered to be a crucial indicator of exercise tolerance in chronic obstructive pulmonary disease patients, which is in significant association with mortality [11]. In this study, 6MWT was examined to assess functional walking ability as well as the treatment response of sequential breathing rehabilitation training of chronic obstructive pulmonary disease. Our results suggested that sequential breathing training can increase the patient's six-minute walking distance, thereby improving their exercise tolerance.

In summary, sequential breathing training strategy for chronic obstructive pulmonary disease is not only limited to the rehabilitation of patients' lung function, but can also be extended to improve the patient's motor function and quality of life, which is worthy of clinical application.

\section{Conclusion}

Our findings revealed that sequential breathing training therapy can distinctly improve $\mathrm{FEVI \% ,} \mathrm{mMRC} \mathrm{score,} \mathrm{CAT} \mathrm{score,} \mathrm{the} \mathrm{number}$ of acute exacerbation frequency and 6MWT for stable chronic obstructive pulmonary disease compared to basic routine treatment. Thus, sequential breathing training therapy can better improve the pulmonary function as well as acute exacerbation incidence for stable chronic obstructive pulmonary disease patients, which is worthy of further clinical exploration.

\section{Declarations}

\section{Acknowledgements}

Not applicable.

\section{Funding}

This work was funded by the Scientific Research Project in Higher Education of Zhejiang (Y201225397); Science and Technology Project of Jinhua (2014A33191).

\section{Availability of data and material}

The datasets analyzed during the current study are available from the corresponding author on reasonable request.

\section{Authors' contributions}

Mingxing Ding conceived and designed the study. Xiaobing Wang, An Guo conducted most of the experiments and data analysis, and wrote the manuscript. Honggang Wang, Wei Huang participated in collecting data and helped to draft the manuscript. All authors reviewed and approved the manuscript.

\section{Ethics approval and consent to participate}

The study was approved by the Ethics Committee of School of Medicine, Jinhua Polytechnic (2014035).

\section{Consent for publication}

All subjects were informed consent.

\section{Conflicts of Interest}

The authors declare no conflicts of interest.

\section{References}

1. Barnes PJ, Burney PG, Silverman EK (2015) Chronic obstructive pulmonary disease. Nat Rev Dis Primers 1:15076. [Crossref]

2. Rosenberg SR, Kalhan R, Mannino DM (2015) Epidemiology of Chronic Obstructive Pulmonary Disease: Prevalence, Morbidity, Mortality, and Risk Factors. Semin Respir Crit Care Med 36: 457-469. [Crossref]

3. Caramori G, Ruggeri P, Arpinelli F (2019) Long-term use of inhaled glucocorticoids in patients with stable chronic obstructive pulmonary disease and risk of bone fractures: a narrative review of the literature. Int J Chron Obstruct Pulmon Dis 14: 1085-1097. [Crossref]

4. Lee HW, Park J, Jo J (2019) Comparisons of exacerbations and mortality among regular inhaled therapies for patients with stable chronic obstructive pulmonary disease: Systematic review and Bayesian network meta-analysis. PLoS Med 16: e1002958. [Crossref]

5. Leong P, Macdonald MI, Ko BS, Bardin PG (2019) Coexisting chronic obstructive pulmonary disease and cardiovascular disease in clinical practice: a diagnostic and therapeutic challenge. Med J Aust 210: 417-423. [Crossref]

6. Ragaselvi S, Janmeja AK, Aggarwal D (2019) Predictors of response to pulmonary rehabilitation in stable chronic obstructive pulmonary disease patients: A prospective cohort study. J Postgrad Med 65: 101-106. [Crossref]

7. Gendron LM, Nyberg A, Saey D (2018) Active mind-body movement therapies as an adjunct to or in comparison with pulmonary rehabilitation for people with chronic obstructive pulmonary disease. Cochrane Database Syst Rev 10: Cd012290. [Crossref]

8. O'Connor C, Lawson R, Waterhouse J, Mills GH (2019) Is inspiratory muscle training (IMT) an acceptable treatment option for people with chronic obstructive pulmonary disease (COPD) who have declined pulmonary rehabilitation (PR) and can IMT enhance PR uptake? A single-group prepost feasibility study in a home-based setting. BMJ Open 9: e028507. [Crossref]

9. Nellessen A, Hernandes NA, Pitta F (2013) Physiotherapy and rehabilitative interventions in patients with chronic respiratory diseases: exercise and non-exercise treatment. Panminerva Med 55: 197-209. [Crossref]

10. de Roos P, Lucas C, Strijbos JH, van Trijffel E (2018) Effectiveness of a combined exercise training and home-based walking programme on physical activity compared with standard medical care in moderate COPD: a randomised controlled trial. Physiotherapy 104: 116-121. [Crossref]

11. Beaumont M, Losq A, Péran L (2019) Comparison of 3-minute Step Test (3MStepT) and 6-minute Walk Test (6MWT) in Patients with COPD. Copd 16: 266-271. [Crossref]

12. Yohannes AM, Dryden S, Hanania NA (2019) The responsiveness of the Manchester Chronic Obstructive Pulmonary Disease Fatigue Scale to pulmonary rehabilitation. Ther Adv Chronic Dis 10: 2040622319882206. [Crossref]

13. Burge AT, Cox NS, Abramson MJ, Holland AE (2020) Interventions for promoting physical activity in people with chronic obstructive pulmonary disease (COPD). Cochrane Database Syst Rev 4: Cd012626. [Crossref]

14. Wang J, Guo S, Zeng M (2019) Observation of the curative effect of device-guided rehabilitation on respiratory function in stable patients with chronic obstructive pulmonary disease. Medicine (Baltimore) 98: e14034. [Crossref]

15. Zheng W, Li M, Hong Y (2019) Traditional Chinese exercise (TCE) on pulmonary rehabilitation in patients with stable chronic obstructive pulmonary disease: Protocol for a systematic review and network meta-analysis. Medicine (Baltimore) 98: e16299. [Crossref]

16. Grassion L, Gonzalez-Bermejo J (2017) Sleep and Mechanical Ventilation in Stable COPD Patients. Copd 14: 411-417. [Crossref] 
17. He M, Yu S, Wang L (2015) Efficiency and safety of pulmonary rehabilitation in acute exacerbation of chronic obstructive pulmonary disease. Med Sci Monit 2015; 21: 806812. [Crossref]

18. Stringer W, Marciniuk D (2018) The Role of Cardiopulmonary Exercise Testing (CPET) in Pulmonary Rehabilitation (PR) of Chronic Obstructive Pulmonary Disease (COPD) Patients. Copd 15: 621-631. [Crossref]

19. Gloeckl R, Schneeberger T, Jarosch I, Kenn K (2018) Pulmonary Rehabilitation and Exercise Training in Chronic Obstructive Pulmonary Disease. Dtsch Arztebl Int 115: 117-123. [Crossref]

20. O'Dell A, Diegel-Vacek L, Burt L, Corbridge S (2018) CE: Managing Stable COPD: An Evidence-Based Approach. Am J Nurs 118: 36-47. [Crossref]

21. Kubincová A, Takáč P, Kendrová L (2018) The Effect of Pulmonary Rehabilitation in Mountain Environment on Exercise Capacity and Quality of Life in Patients with Chronic Obstructive Pulmonary Disease (COPD) and Chronic Bronchitis. Med Sci Monit 24: 6375-6386. [Crossref]

22. Laurent H, Galvaing G, Thivat E (2017) Effect of an intensive 3-week preoperative home rehabilitation programme in patients with chronic obstructive pulmonary disease eligible for lung cancer surgery: a multicentre randomised controlled trial. BMJ Open 7: e017307. [Crossref]

23. Rugbjerg M, Iepsen UW, Jørgensen KJ, Lange P (2015) Effectiveness of pulmonary rehabilitation in COPD with mild symptoms: a systematic review with meta-analyses. Int J Chron Obstruct Pulmon Dis 2015; 10:791-801. [Crossref]
24. Coquart JB, Le Rouzic O, Racil G (2017) Real-life feasibility and effectiveness of homebased pulmonary rehabilitation in chronic obstructive pulmonary disease requiring medical equipment. Int J Chron Obstruct Pulmon Dis 2017; 12:3549-3556. [Crossref]

25. Cheng SL, Lin CH, Wang CC (2019) Comparison between COPD Assessment Test (CAT) and modified Medical Research Council (mMRC) dyspnea scores for evaluation of clinical symptoms, comorbidities and medical resources utilization in COPD patients. J Formos Med Assoc 118: 429-435. [Crossref]

26. Huang WC, Wu MF, Chen HC, Hsu JY (2015) Features of COPD patients by comparing CAT with mMRC: a retrospective, cross-sectional study. NPJ Prim Care Respir Med 25: 15063. [Crossref]

27. Miravitlles M, Koblizek V, Esquinas C (2019) Determinants of CAT (COPD Assessment Test) scores in a population of patients with COPD in central and Eastern Europe: The POPE study. Respir Med 150: 141-148. [Crossref]

28. Pulido Herrero E, García Gutiérrez S, Antón Ladislao A (2019) Chronic obstructive pulmonary disease assessment test: usefulness for monitoring recovery and predicting poor course of disease after exacerbations. Emergencias 31: 21-26. [Crossref]

29. Tsiligianni I, Kampouraki M, Ierodiakonou D (2019) COPD patients' characteristics, usual care, and adherence to guidelines: the Greek UNLOCK study. Int $J$ Chron Obstruct Pulmon Dis 14: 547-556. [Crossref]

30. Zeng GS, Chen LC, Fan HZ (2019) The relationship between steps of 6MWT and COPD severity: a cross-sectional study. Int J Chron Obstruct Pulmon Dis 14: 141-148. [Crossref]

31. Gulart AA, Munari AB, Queiroz AP (2017) Does the COPD assessment test reflect functional status in patients with COPD? Chron Respir Dis 14: 37-44. [Crossref]

Copyright: @2020 Wang X. This is an open-access article distributed under the terms of the Creative Commons Attribution License, which permits unrestricted use, distribution, and reproduction in any medium, provided the original author and source are credited. 